\title{
Strong convergence theorems for k-strictly pseudo-contractive mapping in Hilbert spaces
}

\author{
Qiuxia $\mathrm{Lu}^{1, \mathrm{a}}$, Yan $\mathrm{Hao}^{2, \mathrm{~b}}$ \\ ${ }^{1,2}$ School of Mathematics, Physics and Information Science, Zhejiang Ocean University, \\ Key Laboratory of Oceanographic Big Data Mining \& Application of Zhejiang Province, \\ Zhoushan, Zhejiang 316022, China \\ a535645272@qq.com, bhaoyanmao@126.com
}

Keywords: Fixed point; Iterative method; $k$-strictly pseudo-contractive mapping

Abstract: In this paper, we introduce a general iterative algorithm and prove strong convergence theorems for a non-self k-strictly pseudo-contractive mappings in Hilbert spaces. Our results improve and extend the corresponding results announced by many others.

\section{Introduction and Preliminaries}

Let $K$ be a nonempty subset of a Hilbert space $H$. Recall that a mapping $T: K \rightarrow H$ is said to be a $k$-strictly pseudo-contractive if there exists a constant $k \in[0,1)$ such that

$$
\|T x-T y\|^{2} \leq\|x-y\|^{2}+k\|(I-T) x-(I-T) y\|^{2} \text { for all } x, y \in K \text {. }
$$

Note that the class of $k$-strictly pseudo-contractions includes strictly the class of nonexpansive mapping which are mappings $T$ on $K$ such that

$$
\|T x-T y\| \leq\|x-y\|, \forall x, y \in K \text {. }
$$

That is, $T$ is nonexpansive if and only if $T$ is 0 -strictly pseudo-contractive.

In 2002, Marino and $\mathrm{Xu}^{[1]}$ introduced and considered the following iterative algorithm:

$$
\left\{\begin{array}{l}
x_{0} \in K, \\
x_{n+1}=\alpha_{n} \gamma f\left(x_{n}\right)+\left(I-\alpha_{n} A\right) T x_{n}, \forall n \geq 0 .
\end{array}\right.
$$

Theorem MX. Let $H$ be a Hilbert space, $K$ be a closed convex subset of $H, T: K \rightarrow K$ be a nonexpansive mapping with $F(T) \neq \varnothing$. Let $A$ be a strong positive bounded linear operator on $K$ with coefficient $\bar{\gamma}$ and $f: K \rightarrow K$ be a contraction with the contractive coefficient $(0<\alpha<1)$ such that $0<\gamma<\frac{\bar{\gamma}}{\alpha}$. Let $\left\{x_{n}\right\}$ be a sequence in $K$ generated by (1.3). Then, under the hypotheses (i) $\lim _{n \rightarrow \infty} \alpha_{n}=0$, (ii) $\sum_{n=1}^{\infty} \alpha_{n}=\infty$ and (iii) either $\sum_{n=1}^{\infty}\left|\alpha_{n+1}-\alpha_{n}\right|<\infty$ or $\lim _{n \rightarrow \infty} \frac{\alpha_{n+1}}{\alpha_{n}}=1$, $\left\{x_{n}\right\}$ converges strongly to a fixed point $q$ of $T$, which is the unique solution of the following variational inequality related to the linear operator $A$ :

$$
\langle(A-\gamma f) q, q-p\rangle \leq 0, \forall p \in F(T) \text {. }
$$

In this paper, motivated by Marino and $\mathrm{Xu}$, we introduce a general iterative and prove strong convergence theorems for $k$-strictly pseudo-contractive mappings in Hilbert spaces. Our results improve and extend the corresponding ones announced by many others.

Throughout this paper, we use $F(T)$ to denote the fixed point set of the mapping $T$ and $P_{K}$ to denote the metric projection of a Hilbert space $H$ onto a closed convex subset $K$ of $H$. Recall that a self-mapping $f: K \rightarrow K$ is a contraction on $K$ if there exists a constant $\alpha \in(0,1)$ such that

$$
\|f(x)-f(y)\| \leq \alpha\|x-y\|, \forall x, y \in K .
$$

In order to prove our main results, we need the following definitions and lemmas. 
Lemma $1.1^{[2]}$ If $T$ is a $k$-strictly pseudo-contraction on a closed convex subset of $K$ of a real Hilbert space $H$, then the fixed point set $F(T)$ is closed convex so that the projection $P_{F(T)}$ is well defined.

Lemma $1.2^{[2]}$ Let $H$ be a Hilbert space, $K$ be a closed convex subset of $H$. Let $T: K \rightarrow H$ be a $k$-strictly pseudo-contractive mapping with $F(T) \neq \varnothing$. Then $F\left(P_{K} T\right)=F(T)$.

Lemma $1.3^{[2]}$ Let $T: K \rightarrow H$ be a $k$-strictly pseudo-contraction. Define $S: K \rightarrow H$ by $S x=\lambda x+(1-\lambda) T x$ for each $x \in K$.Then, as $\lambda \in[k, 1), S$ is a nonexpansive mapping such that $F(S)=F(T)$.

Lemma 1.4 ${ }^{[3]}$ Assume that $\left\{\alpha_{n}\right\}$ is a sequence of nonnegative real numbers such that $\alpha_{n+1} \leq\left(1-\gamma_{n}\right) \alpha_{n}+\delta_{n}, \forall n \geq 0$. where $\left\{\gamma_{n}\right\}$ is a sequence in $(0,1)$ and $\left\{\delta_{n}\right\}$ is a sequence such that (i) $\sum_{n=1}^{\infty} \gamma_{n}=\infty$; (ii) $\limsup \sup _{n \rightarrow \infty} \frac{\delta_{n}}{\gamma_{n}} \leq 0$ or $\sum_{n=1}^{\infty}\left|\delta_{n}\right|<\infty$, Then $\lim _{n \rightarrow \infty} \alpha_{n}=0$.

Lemma $1.5^{[4]}$. Let $H$ be a real Hilbert space, the following inequality holds $\|x+y\|^{2} \leq\|x\|^{2}+2\langle y, x+y\rangle, \forall x, y \in H$.

\section{Main results}

Theorem2.1. Let $K$ be a nonempty closed convex subset of a real Hilbert space $H$ and $T: K \rightarrow H$ be a $k$-strictly pseudo-contractive mapping with a common fixed point for some $0 \leq k<1$. Let $f: K \rightarrow K$ be a contraction with the contractive coefficient $(0<\alpha<1)$. Let $\left\{x_{n}\right\}$ be a sequence in $K$ generated in the following manner:

$$
\left\{\begin{array}{l}
x_{1} \in K, \\
x_{n+1}=\alpha_{n} f\left(x_{n}\right)+\left(1-\alpha_{n}\right) P_{K} S x_{n}, \forall n \geq 1 .
\end{array}\right.
$$

where $S: K \rightarrow H$ is a mapping defined by $S x=\lambda x+(1-\lambda) T x$. If the control sequence $\left\{\alpha_{n}\right\}$ satisfies the following conditions: (i) $\lim _{n \rightarrow \infty} \alpha_{n}=0$; (ii) $\sum_{n=1}^{\infty} \alpha_{n}=\infty$; (iii) $\sum_{n=1}^{\infty}\left|\alpha_{n+1}-\alpha_{n}\right|<\infty$. Then $\left\{x_{n}\right\}$ converges strongly to a fixed point $q$ of $T$, which solves the following variational inequality:

$$
\langle f(q)-q, p-q\rangle \leq 0, \forall p \in F(T) \text {. }
$$

Proof. From Lemma1.3, we know that the mapping $S: K \rightarrow H$ is a nonexpansive mapping and $F(S)=F(T)$, By our assumptions on $T$, we have $F(T) \neq \varnothing$. By Lemma1.1, we see $F\left(P_{K} S\right)=F(S) \neq \varnothing$. Since $P_{K}: H \rightarrow K$ is a nonexpansive mapping, we conclude that $P_{K} S: K \rightarrow K$ is also nonexpansive. Observing the condition (i), we may assume that $\alpha_{n}<1$ for all $n \geq 1$. Taking a point $p \in F(T)$, we obtain

$$
\begin{aligned}
\left\|x_{n+1}-p\right\| & =\left\|\alpha_{n}\left(f\left(x_{n}\right)-p\right)+\left(1-\alpha_{n}\right)\left(P_{K} S x_{n}-p\right)\right\| \\
& \leq\left(1-\alpha_{n}\right)\left\|P_{K} S x_{n}-p\right\|+\alpha_{n}\left\|f\left(x_{n}\right)-p\right\| \\
& \leq\left[1-\alpha_{n}(1-\alpha)\right]\left\|x_{n}-p\right\|+\alpha_{n}\|f(p)-p\| .
\end{aligned}
$$

By simple inductions, we have $\left\|x_{n}-p\right\| \leq \max \left\{\left\|x_{0}-p\right\|, \frac{\|p-f(p)\|}{1-\alpha}\right\}, \forall n \geq 1$,

which yields that the sequence $\left\{x_{n}\right\}$ is bounded. On the other hand, we have

$$
\begin{aligned}
x_{n+2}-x_{n+1}= & \left(1-\alpha_{n}\right)\left(P_{K} S x_{n+1}-P_{K} S x_{n}\right)-\left(\alpha_{n+1}-\alpha_{n}\right) P_{K} S x_{n} \\
& +\left[\alpha_{n+1}\left(f\left(x_{n+1}\right)-f\left(x_{n}\right)\right)+f\left(x_{n}\right)\left(\alpha_{n+1}-\alpha_{n}\right)\right] .
\end{aligned}
$$

which yields that 


$$
\begin{aligned}
\left\|x_{n+2}-x_{n+1}\right\| \leq & \left(1-\alpha_{n+1}\right)\left\|x_{n+1}-x_{n}\right\|+\left|\alpha_{n+1}-\alpha_{n}\right|\left\|P_{K} S x_{n}\right\| \\
& +\left[\alpha_{n+1} \alpha\left\|x_{n+1}-x_{n}\right\|\right]+\left\|f\left(x_{n}\right)\right\|\left\|\alpha_{n+1}-\alpha_{n}\right\| \\
& \leq\left[1-\alpha_{n+1}(1-\alpha)\right]\left\|x_{n+1}-x_{n}\right\|+\left|\alpha_{n+1}-\alpha_{n}\right| M_{1},
\end{aligned}
$$

where $M_{1}$ is an appropriate constant such that $M_{1} \geq \sup _{n \geq 1}\left\{\left\|P_{K} S x_{n}\right\|+\left\|f\left(x_{n d}\right)\right\|\right\}$.

Noticing the condition (i), (ii) and (iii) and apply Lemma (1.4) to (2.1),

we have $\lim _{n \rightarrow \infty}\left\|x_{n+1}-x_{n}\right\|=0$.

Notice that $\left\|x_{n}-P_{K} S_{n}\right\| \leq\left\|x_{n}-x_{n+1}\right\|+\left\|x_{n+1}-P_{K} S x_{n}\right\| \leq\left\|x_{n+1}-x_{n}\right\|+\alpha_{n}\left\|f\left(x_{n}\right)-P_{K} S x_{n}\right\|$.

It follows from the condition (i) and (2.2) that $\lim _{n \rightarrow \infty}\left\|x_{n}-P_{K} S x_{n}\right\|=0$.

Next we claim that $\lim \sup \left\langle f(q)-q, x_{n}-q\right\rangle \leq 0$,

where $q=\lim _{t \rightarrow 0} x_{t}$ with $x_{t}$ being the fixed point of the contraction $x$ a $t f(x)+(1-t) P_{K} S x$.

Then $x_{t}$ solves the fixed point equation $x_{t}=t f\left(x_{t}\right)+(1-t) P_{K} S x_{t}$. Thus we have

$$
\left\|x_{t}-x_{n}\right\|=\left\|(1-t)\left(P_{K} S x_{t}-x_{n}\right)+t\left(f\left(x_{t}\right)-x_{n}\right)\right\| .
$$

It follows from the Lemma 1.5 that

$$
\begin{aligned}
\left\|x_{t}-x_{n}\right\|^{2} & =\left\|(1-t)\left(P_{K} S x_{t}-x_{n}\right)+t\left(f\left(x_{t}\right)-x_{n}\right)\right\|^{2} \\
& \leq(1-t)^{2}\left\|P_{K} S x_{t}-x_{n}\right\|^{2}+2 t\left\langle f\left(x_{t}\right)-x_{n}, x_{t}-x_{n}\right\rangle \\
& \leq\left(1-2 t+t^{2}\right)\left\|x_{t}-x_{n}\right\|^{2}+f_{n}(t)+2 t\left\langle f\left(x_{t}\right)-x_{t}, x_{t}-x_{n}\right\rangle+2 t\left\langle x_{t}-x_{n}, x_{t}-x_{n}\right\rangle,
\end{aligned}
$$

where $f_{n}(t)=\left(2\left\|x_{t}-x_{n}\right\|+\left\|x_{n}-P_{K} S x_{n}\right\|\right)\left\|x_{n}-P_{K} S x_{n}\right\| \rightarrow 0(n \rightarrow \infty)$.

and $\left\langle x_{t}-x_{n}, x_{t}-x_{n}\right\rangle=\left\|x_{t}-x_{n}\right\|^{2}$.

Combining (2.5) and (2.7), we have

$$
\begin{gathered}
2 t\left\langle x_{t}-f\left(x_{t}\right), x_{t}-x_{n}\right\rangle \leq\left(t^{2}-2 t\right)\left\|x_{t}-x_{n}\right\|^{2}+f_{n}(t)+2 t\left\langle x_{t}-x_{n}, x_{t}-x_{n}\right\rangle \\
\leq t^{2}\left\|x_{t}-x_{n}\right\|^{2}+f_{n}(t)
\end{gathered}
$$

It follows that $\left\langle x_{t}-f\left(x_{t}\right), x_{t}-x_{n}\right\rangle \leq \frac{t}{2}\left\|x_{t}-x_{n}\right\|^{2}+\frac{1}{2 t} f_{n}(t)$.

Letting $n \rightarrow \infty$ in (2.8) and noting (2.6) yields

$$
\limsup _{n \rightarrow \infty}\left\langle x_{t}-f\left(x_{t}\right), x_{t}-x_{n}\right\rangle \leq \frac{t}{2} M_{2} \text {, }
$$

where $M_{2}>0$ is a constant such that $M_{2} \geq\left\|x_{t}-x_{n}\right\|^{2}$ for all $t \in(0,1)$ and $n \geq 1$. Taking $t \rightarrow 0$ in (2.9), we have $\limsup _{t \rightarrow 0} \limsup _{n \rightarrow \infty}\left\langle x_{t}-f\left(x_{t}\right), x_{t}-x_{n}\right\rangle \leq 0$.

On the other hand, we have

It follows that

$$
\begin{aligned}
\left\langle f(q)-q, x_{n}-q\right\rangle= & \left\langle f(q)-q, x_{n}-q\right\rangle-\left\langle f(q)-q, x_{n}-x_{t}\right\rangle \\
& +\left\langle f(q)-q, x_{n}-x_{t}\right\rangle-\left\langle f(q)-x_{t}, x_{n}-x_{t}\right\rangle \\
& +\left\langle f(q)-x_{t}, x_{n}-x_{t}\right\rangle-\left\langle f\left(x_{t}\right)-x_{t}, x_{n}-x_{t}\right\rangle+\left\langle f\left(x_{t}\right)-x_{t}, x_{n}-x_{t}\right\rangle .
\end{aligned}
$$

$$
\limsup _{n \rightarrow \infty}\left\langle f(q)-q, x_{n}-q\right\rangle \leq\|f(q)-q\|\left\|x_{t}-q\right\|+\left\|x_{t}-q\right\| \lim _{n \rightarrow \infty}\left\|x_{n}-x_{t}\right\|
$$




$$
+\alpha\left\|q-x_{t}\right\| \lim _{n \rightarrow \infty}\left\|x_{n}-x_{t}\right\|+\limsup _{n \rightarrow \infty}\left\langle f\left(x_{t}\right)-x_{t}, x_{n}-x_{t}\right\rangle .
$$

Therefore, from(2.10), it follows that

$$
\limsup _{n \rightarrow \infty}\left\langle f(q)-q, x_{n}-q\right\rangle=\limsup _{t \rightarrow 0} \limsup _{n \rightarrow \infty}\left\langle f(q)-q, x_{n}-q\right\rangle \leq 0 .
$$

Hence (2.4) holds. Now from the Lemma 1.5, we have

$$
\begin{aligned}
\left\|x_{n+1}-q\right\|^{2} & =\left\|\left(1-\alpha_{n}\right)\left(P_{K} S x_{n}-q\right)+\alpha_{n}\left(f\left(x_{n}\right)-q\right)\right\|^{2} \\
& \leq\left(1-\alpha_{n}\right)^{2}\left\|x_{n}-q\right\|^{2}+\alpha_{n} \alpha\left(\left\|x_{n}-q\right\|^{2}+\left\|x_{n+1}-q\right\|^{2}\right)+2 \alpha_{n}\left\langle f(q)-q, x_{n+1}-q\right\rangle,
\end{aligned}
$$

which implies that

$$
\begin{aligned}
& \left\|x_{n+1}-q\right\|^{2} \leq \frac{\left(1-\alpha_{n}\right)^{2}+\alpha_{n} \alpha}{1-\alpha_{n} \alpha}\left\|x_{n}-q\right\|^{2}+\frac{2 \alpha_{n}}{1-\alpha_{n} \alpha}\left\langle f(q)-q, x_{n+1}-q\right\rangle \\
& \leq\left[1-\frac{2 \alpha_{n}(1-\alpha)}{1-\alpha_{n} \alpha}\right]\left\|x_{n}-q\right\|^{2}+\frac{2 \alpha_{n}(1-\alpha)}{1-\alpha_{n} \alpha}\left[\frac{1}{1-\alpha}\left\langle f(q)-q, x_{n+1}-q\right\rangle+\frac{\alpha_{n}}{2(1-\alpha)} M_{3}\right]
\end{aligned}
$$

where $M_{3}$ is an appropriate constant such that $M_{3} \geq \sup _{n \geq 1}\left\{\left\|x_{n}-q\right\|^{2}\right\}$.

Put $j_{n}=\frac{2 \alpha_{n}(1-\alpha)}{1-\alpha_{n} \alpha}$ and $t_{n}=\frac{1}{1-\alpha}\left\langle f(q)-q, x_{n+1}-q\right\rangle+\frac{\alpha_{n}}{2(1-\alpha)} M_{3}$.

Then we have $\left\|x_{n+1}-q\right\|^{2} \leq\left(1-j_{n}\right)\left\|x_{n}-q\right\|+j_{n} t_{n}$.

It follows from the conditions (i),(ii)and(2.4) that $\lim _{n \rightarrow \infty} j_{n}=0, \sum_{n=1}^{\infty} j_{n}=\infty, \limsup _{n \rightarrow \infty} t_{n} \leq 0$.

Therefore, applying Lemma1.4 to(2.13), we have $x_{n} \rightarrow q$ as $n \rightarrow \infty$. This completes the proof.

\section{References}

[1] H.K. Xu, Another control condition in an iterative method for nonexpansive mappings, Bull. Austral. Math. Soc. 65 (2002) 109-113.

[2] H.K. Xu, An iterative approach to quadratic optimization, J. Optim. Theory Appl. 116 (2003) 659-678.

[3] B.E. Rhoades, Fixed point iterations using infinite matrices, Trans. Amer. Math. Soc. 196 (1974) 162-176.

[4] S. Reich, Weak convergence theorems for nonexpansive mappings in Banach spaces, J. Math. Anal. Appl. 67 (1979) 274-276. 